\title{
The impact of artificial intelligence in screening for diabetic retinopathy in India
}

\author{
Ramachandran Rajalakshmi ${ }^{1}$
}

Received: 20 September 2019 / Accepted: 25 September 2019 / Published online: 11 December 2019

(c) The Royal College of Ophthalmologists 2019

Diabetic retinopathy (DR), a morbid microvascular complication of diabetes, affects one in three people with diabetes and is one of the leading causes of preventable blindness. With over 400 million people with diabetes worldwide, the overall prevalence of DR is $34.6 \%$, with $10.2 \%$ of them having sight-threatening diabetic retinopathy (STDR) [1]. India is home to over 72 million people with diabetes with an estimated DR prevalence of around $18 \%$ [2].

As people with diabetes may not have visual symptoms, they may not visit the ophthalmologist for regular retinal screening. Their regular point of care would be the endocrinology/diabetology or the physician clinic. The clinical course of DR has a long asymptomatic stage and hence the importance of screening is well established for early detection, as timely intervention can reduce visual impairment due to STDR [3]. Fundus photography has been accepted as a mode of screening for DR with grading of the fundus photographs being done by trained certified graders/ retina specialists. With the exponential increase in the number of people with diabetes, and shortage of trained graders/retinal specialists, an automated approach using artificial intelligence (AI) for DR screening has started attracting attention of healthcare professionals.

$\mathrm{AI}$ is an efficient computer-based tool that augments human intelligence in healthcare, with the use of algorithms in the analysis of complex medical data to provide useful diagnostic outputs. AI and deep learning (DL) find applications in ophthalmology as a large part of data to be analysed are image based and the outcomes are related to image recognition.

Ramachandran Rajalakshmi

drraj@drmohans.com

1 Dr. Mohan's Diabetes Specialities Centre and Madras Diabetes Research Foundation, No. 6, Conransmith Road Gopalapuram, Chennai, Tamilnadu 600086, India
The use of AI in DR screening has made successful inroads with respect to diagnosis and providing referral recommendations. The role of $\mathrm{AI}$ in detection of referable $\mathrm{DR}$ (RDR), which is defined as grade of retinopathy of moderate non-proliferative DR (NPDR) and above, with and without diabetic macular oedema (DME) appear promising with high sensitivity and specificity levels in many studies using various $\mathrm{AI}$ algorithms being over 90\% [4]. AI algorithms need skilled manpower to capture clear and good quality high resolution images to be fed as input images as well as trained optometrists/ophthalmologists/ retina specialists (annotators) to provide accurate ground truth for training the images.

The first published study of use of AI algorithms with smartphone-based fundus images for DR detection was from India. A retrospective analysis of mydriatic smartphone-based retinal images and EyeArt AI software showed a high sensitivity of $95.8 \%$ for detection of DR of any level of severity and over $99 \%$ sensitivity for detection of RDR as well as STDR [5].

The study by Sahlsten et al. showed that a DL system could identify RDR and DR of varying severity as well as DME better when the image quality is good, and best when the images had a high resolution [6]. This study was performed with anonymized retinal images of people from Finland, used the binary classification for DR; NRDR (nonRDR) and RDR and NRDME (non-referable DME) and referable DME. The accuracy was found to be higher than that of some of the other similar studies, despite the training of the DL system being done with lesser number of images. They have shown that they achieved the best results of accuracy while using the largest $2095 \times 2095$ pixels input image size, emphasising that image quality is the key in AI assisted DR diagnosis.

In a nationwide DR screening program in Thailand, a large-scale validation study done to compare the performance of a DL algorithm developed by Google vs the grading by local ophthalmologists/retina specialists showed that the DL algorithm had a high sensitivity and specificity for detection of RDR as well as STDR (severe NPDR/PDR/ 
DME) [7]. The study has shown that real-time implementation of AI for large-scale DR screening is possible. This study is an early milestone in clinical implementation of use of AI in DR screening.

While these studies have demonstrated the ability of AI to detect RDR in retinal colour photographs with high sensitivity and specificity, majority of the earlier studies on the role of $\mathrm{AI}$ in DR were performed with retrospective image data.

Natarajan et al. recently published a prospective study on the real time use of AI in clinical setting, analysing smartphone based retinal images of 231 patients with diabetes visiting dispensaries under the municipality of Greater Mumbai [8]. The study was done using an offline AI algorithm (Medios AI) that could be used on a smartphone, to detect RDR with retinal colour photographs taken on Fundus on Phone (FOP NM-10). The results showed very high sensitivity $(100 \%)$ and specificity $(88.4 \%)$ in grading RDR, when compared with manual grading by trained ophthalmologists.

The short time taken, accuracy, consistency, and scalability are the major advantages that make the role of AI in DR detection appear promising. Majority of the AI studies published so far have looked mainly at two-dimensional colour fundus images for DR screening. Future publications that would assess the role of $\mathrm{AI}$ in multimodal imaging in DR would be looked at with keen interest.

From the above studies, it appears that real-time deployment of AI in screening for DR is feasible. It fits in with the current trend of telemedicine for screening and automated retinal image analysis extends opportunities to screening in more remote areas. AI assisted DR algorithms could potentially expedite early detection of DR at the primary care level and would be helpful in screening large numbers of people with diabetes in low and middle income countries like India. The ability of AI algorithms to detect not just DR but other disorders like age-related macular degeneration and glaucoma will increase the utility of AI in ophthalmology.

Though the AI-based systems continue to soar high in terms of potential applications in healthcare and ophthalmology, we need to remember that the basis of diagnoses made by AI algorithms is mechanical, and that human interpretations by ophthalmologists/retina specialists is essential in case of variations and for further management.

\section{Compliance with ethical standards}

Conflict of interest The author declares that he has no conflict of interest.

Publisher's note Springer Nature remains neutral with regard to jurisdictional claims in published maps and institutional affiliations.

\section{References}

1. Yau JW, Rogers SL, Kawasaki R, Lamoureux EL, Kowalski JW, Bek T, et al. Meta-Analysis For Eye Disease (META-EYE) Study Group. Global prevalence and major risk factors of diabetic retinopathy. Diabetes Care. 2012;35:556-64.

2. Rema M, Premkumar S, Anitha B, Deepa R, Pradeepa R, Mohan V. Prevalence of diabetic retinopathy in urban India: The Chennai urban rural epidemiology study (CURES) eye study, I. Investig Ophthalmol Vis Sci. 2005;46:2328-33.

3. Namperumalswamy P, Nirmalan PK, Ramaswamy K. Developing a screening program to detect sight threatening retinopathy in south India. Diabetes Care. 2003;26:1831-35.

4. Raman R, Srinivasan S, Virmani S, Sivaprasad S, Rao C, Rajalakshmi R. Fundus photograph-based deep learning algorithms in detecting diabetic retinopathy. Eye. 2019;33:97-109.

5. Rajalakshmi R, Subashini R, Anjana RM, Mohan V. Automated diabetic retinopathy detection in smartphonebased fundus photography using artificial intelligence. Eye. 2018;32:1138-44.

6. Sahlsten J, Jaskari J, Kivinen J, Turunen L, Jaanio E, Hietala K, et al. Deep learning fundus image analysis for diabetic retinopathy and macular edema grading. Sci Rep. 2019;9:10750. https://doi. org/10.1038/s41598-019-47181-w. PubMed PMID: 31341220.

7. Raumviboonsuk P, Krause J, Chotcomwongse P, Sayres R, Raman $\mathrm{R}$, Widner K, et al. Deep learning versus human graders for classifying diabetic retinopathy severity in a nationwide screening program. NPJ Digit Med. 2019;2:25 https://doi.org/10.1038/ s41746-019-0099-8. eCollection 2019.

8. Natarajan S, Jain A, Krishnan R, Rogye A, Sivaprasad S. Diagnostic accuracy of community-based diabetic retinopathy screening with an offline artificial intelligence system on a smartphone. JAMA Ophthalmol. 2019. https://doi.org/10.1001/jamaophthalmol. 2019.2923. 